One-dimensional exciton luminescence induced by extended defects in nonpolar $\mathrm{GaN} /(\mathrm{Al}, \mathrm{Ga}) \mathrm{N}$ quantum wells

This article has been downloaded from IOPscience. Please scroll down to see the full text article. 2011 Semicond. Sci. Technol. 26025012

(http://iopscience.iop.org/0268-1242/26/2/025012)

View the table of contents for this issue, or go to the journal homepage for more

Download details:

IP Address: 128.178.195.233

The article was downloaded on 05/01/2011 at 09:54

Please note that terms and conditions apply. 


\title{
One-dimensional exciton luminescence induced by extended defects in nonpolar GaN/(Al,Ga)N quantum wells
}

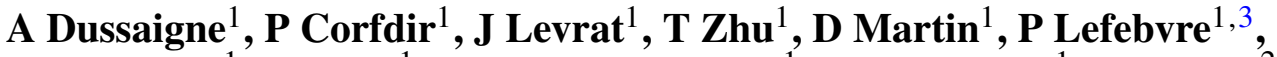 \\ J-D Ganière ${ }^{1}$, R Butté ${ }^{1}$, B Deveaud-Plédran ${ }^{1}$, N Grandjean ${ }^{1}$, Y Arroyo ${ }^{2}$ \\ and P Stadelmann ${ }^{2}$ \\ ${ }^{1}$ ICMP, Ecole Polytechnique Fédérale de Lausanne (EPFL), CH-1015 Lausanne, Switzerland \\ ${ }^{2}$ CIME, Ecole Polytechnique Fédérale de Lausanne (EPFL), CH-1015 Lausanne, Switzerland \\ E-mail: amelie.dussaigne@epfl.ch
}

Received 15 October 2010, in final form 18 October 2010

Published 24 December 2010

Online at stacks.iop.org/SST/26/025012

\begin{abstract}
In this study, we present the optical properties of nonpolar $\mathrm{GaN} /(\mathrm{Al}, \mathrm{Ga}) \mathrm{N}$ single quantum wells (QWs) grown on either $a$ - or $m$-plane $\mathrm{GaN}$ templates for $\mathrm{Al}$ contents set below $15 \%$. In order to reduce the density of extended defects, the templates have been processed using the epitaxial lateral overgrowth technique. As expected for polarization-free heterostructures, the larger the QW width for a given $\mathrm{Al}$ content, the narrower the $\mathrm{QW}$ emission line. In structures with an $\mathrm{Al}$ content set to 5 or $10 \%$, we also observe emission from excitons bound to the intersection of $\mathrm{I}_{1}$-type basal plane stacking faults (BSFs) with the QW. Similarly to what is seen in bulk material, the temperature dependence of BSF-bound QW exciton luminescence reveals intra-BSF localization. A qualitative model evidences the large spatial extension of the wavefunction of these BSF-bound QW excitons, making them extremely sensitive to potential fluctuations located in and away from BSF. Finally, polarization-dependent measurements show a strong emission anisotropy for BSF-bound QW excitons, which is related to their one-dimensional character and that confirms that the intersection between a BSF and a $\mathrm{GaN} /(\mathrm{Al}, \mathrm{Ga}) \mathrm{N}$ QW can be described as a quantum wire.
\end{abstract}

(Some figures in this article are in colour only in the electronic version)

\section{Introduction}

The realization of high radiative efficiency group-III nitridebased quantum wells (QWs) grown along the $c$-axis is currently a key issue to be solved when aiming at increasing the optical power of devices emitting not only in the ultraviolet or the blue ranges of the electromagnetic spectrum, but also in the green one [1,2]. Indeed, the polarization discontinuities at the hetero-interfaces of $c$-plane nitride-based QWs induce a large built-in electric field that decreases the overlap between electron and hole wavefunctions, with detrimental consequences on the room-temperature radiative efficiency.

3 Present address: Instituto de Sistemas Optoelectrnicos y Microtecnologa, ETSI Telecomunicacin, Universidad Politcnica, 28040 Madrid, Spain.
The use of nitride-based heterostructures grown along the

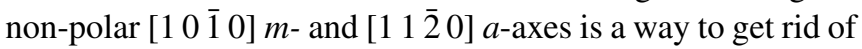
this quantum-confined Stark effect present in polar QWs [3]. Yet, the growth of nonpolar GaN layers is complicated because of adatom incorporation anisotropy between [ $\left[\begin{array}{llll}0 & 0 & 0 & 1\end{array}\right]$ and nonpolar axes [4]. Moreover, nonpolar GaN grown on foreign substrates exhibits high densities of extended defects such as stacking faults [5-8], and a broad defect-related emission line centered at $3.42 \mathrm{eV}$ usually appears to the detriment of the near band-edge emission at $3.47 \mathrm{eV}$ at low temperature $(T<10 \mathrm{~K})$. Whereas this line is seldom observed in $c$-plane $\mathrm{GaN}$ [9-13], it is generally encountered in nonpolar $\mathrm{GaN}$ and has been attributed to the recombination of excitons bound to intrinsic $\mathrm{I}_{1}$-type basal plane stacking faults (BSFs) [14]. Recently, a BSF-related line has also been observed in the 
Table 1. Full-width at half maximum (FWHM), localization energy $\left(E_{\mathrm{loc}}\right)$, and decay time $\left(\tau_{\mathrm{PL}}\right)$ of A and B transitions measured at $10 \mathrm{~K}$ for $a$-plane (Al-content: 5, 10, and 15\%; well width: 2, 4, and $7 \mathrm{~nm}$ ) and $m$-plane samples (Al-content: 5 and $10 \%$; well width: 2,5 , and 8 nm).

\begin{tabular}{|c|c|c|c|c|c|c|c|}
\hline $\begin{array}{l}\text { Template } \\
\text { orientation }\end{array}$ & $\begin{array}{l}\text { Quantum well } \\
\text { width }(\mathrm{nm})\end{array}$ & $\begin{array}{l}\text { Barrier } \mathrm{Al} \\
\text { content }(\%)\end{array}$ & $\begin{array}{l}\text { FWHM A } \\
\text { transition (meV) }\end{array}$ & $\begin{array}{l}E_{\mathrm{loc}} \\
\text { A transition }(\mathrm{meV})\end{array}$ & $\begin{array}{l}E_{\mathrm{loc}} \\
\text { B transition (meV) }\end{array}$ & $\begin{array}{l}\tau_{\mathrm{PL}} \\
( \pm 5 \mathrm{ps})\end{array}$ & $\begin{array}{l}\tau_{\mathrm{PL}} \\
\mathrm{B} \text { transition }( \pm 5 \mathrm{ps})\end{array}$ \\
\hline \multirow[t]{8}{*}{$a$-plane [11-20] } & 2 & 15 & 40 & 9 & - & 118 & - \\
\hline & 4 & 15 & 25 & 24 & - & 27 & 37 \\
\hline & 7 & 15 & 17 & 20 & - & 400 & 450 \\
\hline & 2 & 10 & 35 & 16 & - & 415 & 520 \\
\hline & 4 & 10 & 20 & 17 & - & 305 & 350 \\
\hline & 2 & 5 & 24 & 24 & - & 350 & 375 \\
\hline & 4 & 5 & 22 & 9 & 33 & 405 & 480 \\
\hline & 7 & 5 & 18 & 9 & - & 230 & 330 \\
\hline \multirow[t]{5}{*}{$m$-plane [1-100] } & 2 & 10 & 24 & - & - & - & - \\
\hline & 5 & 10 & 30 & 29 & - & 200 & 470 \\
\hline & 8 & 10 & 29 & - & - & 145 & 190 \\
\hline & 2 & 5 & 30 & - & - & - & - \\
\hline & 8 & 5 & 20 & - & - & - & - \\
\hline
\end{tabular}

emission spectra of $\mathrm{GaN} /(\mathrm{Al}, \mathrm{Ga}) \mathrm{N}$ QWs $[15,16]$ and has been attributed to exciton recombination occurring in regions where the QW is intersected by BSFs.

In this paper, we report a detailed study of the photoluminescence (PL) of nonpolar $\mathrm{GaN} /(\mathrm{Al}, \mathrm{Ga}) \mathrm{N}$ single QWs with various widths and $\mathrm{Al}$ contents. Two types of nonpolar planes, $a$ - and $m$-plane, were investigated in order to show that our results are independent of the nonpolar orientation. In low $\mathrm{Al}$ content QWs, an intense transition lying about $30 \mathrm{meV}$ below the QW emission is related to excitons bound to BSFs crossing the QW. In order to quantitatively characterize the localization of excitons on BSFs, we performed spatially resolved and temperaturedependent PL spectroscopy measurements. By analyzing the polarization of the PL related to intersecting QW and BSFs, we finally evidence the one-dimensional (1D) character of BSF-bound QW excitons.

\section{Experimental details}

Thick ( 4-9 $\mu \mathrm{m})$ non-polar GaN layers have first been grown on $r$ - and $m$-plane sapphire substrates by hydride vapor phase epitaxy (HVPE) (detailed growth parameters are reported in $[17,18])$. In order to improve the structural quality of the samples, we processed the templates using the epitaxial lateral overgrowth (ELO) technique [5-7]. The reduction of the stacking fault density is then evidenced by the increase of near band edge PL emission intensity compared to that of the $3.42 \mathrm{eV}$ band at $T=10 \mathrm{~K}[19,20] . \quad a$ - and $m$-plane $\mathrm{GaN}$ buffer layers were therefore patterned with $200 \mathrm{~nm}$ thick

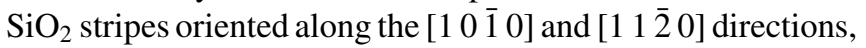
respectively. The 'window' (opening between two stripes) and 'wing' $\left(\mathrm{SiO}_{2}\right.$ stripe) widths are equal to 5 and $45 \mu \mathrm{m}$, respectively. Finally, $\sim 15 \mu \mathrm{m}$ thick $a$-plane or $m$-plane GaN epilayers were overgrown by HVPE.

These templates were then introduced into a Riber compact 21 molecular beam epitaxy system to grow $\mathrm{GaN} /(\mathrm{Al}, \mathrm{Ga}) \mathrm{N}$ single QWs. We used ammonia as a nitrogen source and the different QWs were elaborated using the same growth conditions: $(\mathrm{Al}, \mathrm{Ga}) \mathrm{N}$ growth temperature and growth rate were set to $800-820^{\circ} \mathrm{C}$ and 1 monolayer $\mathrm{s}^{-1}$, respectively. We then deposited a $500 \mathrm{~nm}$ thick $\mathrm{GaN}$ epilayer followed by $200 \mathrm{~nm}$ of $\mathrm{Al}_{x} \mathrm{Ga}_{1-x} \mathrm{~N}$. Various QWs grown on $a$-plane or $m$-plane templates were then prepared in order to study the combined influence of two parameters on the optical properties of the QWs. As shown in table 1, both QW width (values ranging from 2 to $8 \mathrm{~nm}$ ) and $\mathrm{Al}$ content, $x$, (values ranging from $5 \%$ to $15 \%$ ) were varied. Finally, samples were capped with a $20 \mathrm{~nm}$ thick $(\mathrm{Al}, \mathrm{Ga}) \mathrm{N}$ top barrier, of identical $x$ to the bottom one.

The optical characterization of the different structures was performed at low temperature by continuous-wave (cw) PL, micro $(\mu)$ PL and time-resolved (TR) PL. Our cwPL set-up uses the $325 \mathrm{~nm}$ line of a HeCd laser while our $\mu$ PL apparatus uses the $244 \mathrm{~nm}$ line of a frequency-doubled $\mathrm{Ar}^{+}$laser (spatial and spectral resolution of $270 \mathrm{~nm}$ and $0.03 \mathrm{~nm}$, respectively). TRPL was performed using the third harmonic $(\lambda=280 \mathrm{~nm})$ of a pulsed $\mathrm{Ti}: \mathrm{Al}_{2} \mathrm{O}_{3}$ laser (pulse width and repetition rate of 2 ps and $80.7 \mathrm{MHz}$, respectively).

Given the excitation wavelengths and the absorption coefficients of $\mathrm{GaN}$ and $(\mathrm{Al}, \mathrm{Ga}) \mathrm{N}\left(>10^{5} \mathrm{~cm}^{-1}\right)$, we are confident that electron-hole pairs are optically created only within the first 100-200 nm of material from the sample surface. This means that our PL measurements do not directly probe the GaN template or buffer layers, but solely the GaN QWs and their surrounding ( $\mathrm{Al}, \mathrm{Ga}) \mathrm{N}$ barriers. Nevertheless, secondary excitation of the template by the QW luminescence itself is possible.

Polarization analysis of the PL was carried out with a Glan-Thomson polarizer followed by a quarter-wave plate centered at $350 \mathrm{~nm}$. Cross-section transmission electron microscopy (TEM) images were obtained on a Philips CM20 microscope operating at $200 \mathrm{kV}$.

Envelope function calculations were performed using a finite element method with band parameters given in [21]. To treat the case of QW excitons, Coulomb interaction between electron and hole was taken into account by applying the 'effective potential' method [22]. 

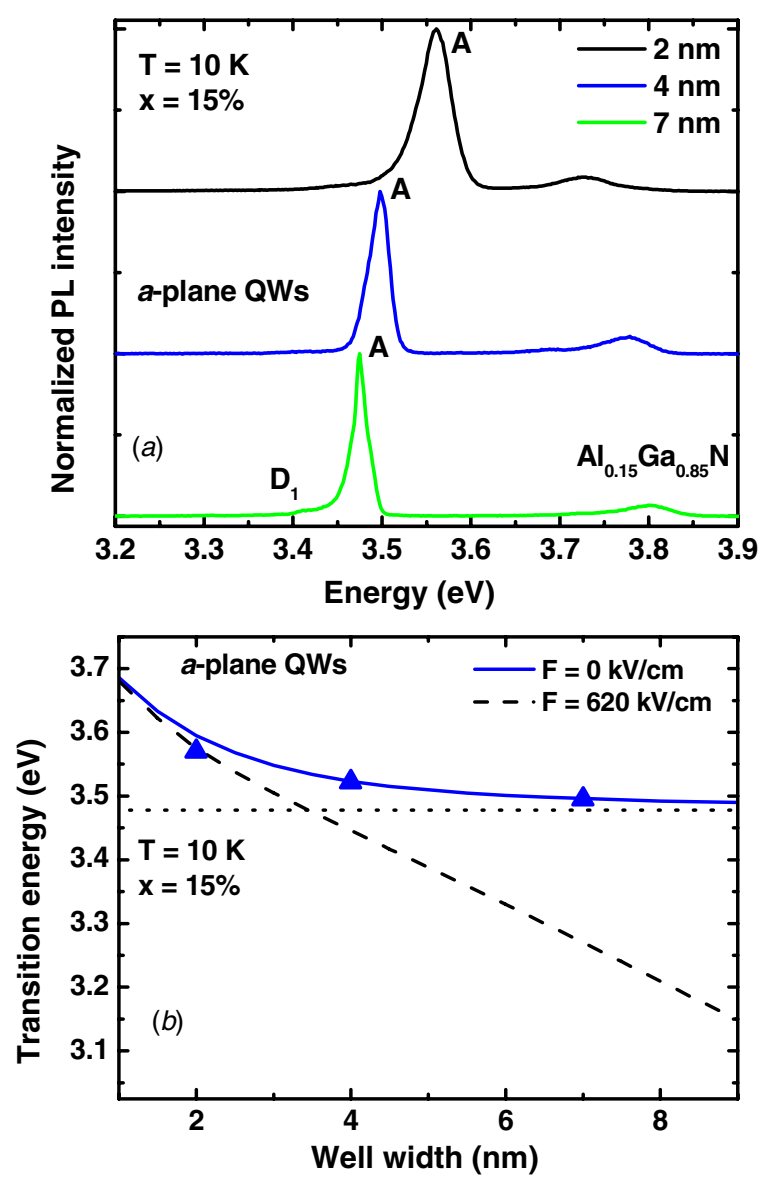

Figure 1. (a) PL spectra measured at $10 \mathrm{~K}$ of $\mathrm{GaN} / \mathrm{Al}_{0.15} \mathrm{Ga}_{0.85} \mathrm{~N}$ QWs of various well widths. Emission lines $A$ and $D_{1}$ are related to the excitonic ground state of the QW and to BSFs present in the $\mathrm{GaN}$ template, respectively. (b) $\mathrm{GaN} / \mathrm{Al}_{0.15} \mathrm{Ga}_{0.85} \mathrm{~N} \mathrm{QW}$ emission energy as a function of well width (symbols). Dashed and solid lines are the result of envelope function calculations with $(620 \mathrm{kV}$ $\left.\mathrm{cm}^{-1}[45]\right)$ and without an internal electric field. Emission energy of free excitons is also reported (black dotted line).

\section{Results and discussion}

\subsection{Quantum well emission (A transition)}

3.1.1. Higher Al content $Q W s(x>15 \%)$. Figure 1(a) shows the PL spectra measured at $10 \mathrm{~K}$ of $a$-plane QWs with an $\mathrm{Al}$ content of $15 \%$. All spectra exhibit a dominant emission peak, labeled $\mathrm{A}$, that we assign to the recombination of the ground state QW exciton. Its emission energy indeed follows the well-width dependence expected from envelope function calculations performed for a polarizationfree single $\mathrm{GaN} / \mathrm{Al}_{0.15} \mathrm{Ga}_{0.85} \mathrm{~N} \mathrm{QW}$ (figure $1(b)$ ). At such low temperatures, the observed excitons are most certainly localized along the QW plane by well-width fluctuations of one or two monolayers [23], as well as by local variations of the $\mathrm{Al}$ composition [24]. If the in-plane extension of such potential fluctuations is small compared with the exciton Bohr radius, the subsequent variations of localization schemes induce variations of emission energies, explaining why the emission linewidth is increased when the well width is reduced. Note that for narrow wells, the penetration of the electron and hole wavefunctions into the disordered alloy barriers also accounts for the inhomogeneous broadening of the PL line (linewidth $\sim 40 \mathrm{meV}$, see table 1).

The higher-energy PL band (3.72-3.8 eV) is attributed to the recombination of excitons localized at potential fluctuations in the $\mathrm{Al}_{x} \mathrm{Ga}_{1-x} \mathrm{~N}$ barriers. One can also observe a weaker band centered at $3.42 \mathrm{eV}$, labeled $\mathrm{D}_{1}$, which is commonly ascribed to excitons bound to $\mathrm{I}_{1}$-BSFs occurring in the GaN template [14].

3.1.2. Lower Al content $Q W s(x<10 \%)$. Thanks to reduced potential fluctuations due to alloy disorder in the barriers, lower $\mathrm{Al}$ content QWs generally present PL spectra with sharper structures [25]. Figure 2(a) displays the low-temperature PL spectra of $\mathrm{GaN} / \mathrm{Al}_{0.1} \mathrm{Ga}_{0.9} \mathrm{~N} a$-plane QWs. Two transitions are now clearly resolved for all samples with $\mathrm{Al}$ contents set to 5 and $10 \%$ (for the sake of clarity, we only report the PL spectra for $\mathrm{GaN} / \mathrm{Al}_{0.1} \mathrm{Ga}_{0.9} \mathrm{~N} a$-plane QWs). According to our envelope function simulations, we attribute the high-energy transition to the emission of the QW excitonic ground state (A transition). The experimental and calculated QW ground state emission energy as a function of QW width are reported in figure 2(b) for $\mathrm{Al}$ contents of 5, 10 and $15 \%$. For $2 \mathrm{~nm}$ thick QWs and Al content higher than 5\%, we note that experimental energies are lower than what is expected from calculations. We believe that such a red-shift results from an enhanced localization effect in regions of lower $\mathrm{Al}$ concentration, thus of lower energy. Indeed, due to the higher penetration of their wavefunction into the barriers, excitons in $2 \mathrm{~nm}$ thick QWs are more sensitive to barrier alloy fluctuations than in wider wells. We suggest that somewhat large Al composition fluctuations in the present samples could be related to $\mathrm{Al}$ adatom incorporation anisotropy due to growth on nonpolar planes. It is also confirmed by a larger linewidth (see table 1).

Similarly to the A transition, the lower energy peak (B transition) red-shifts when the well width increases (figure 2(c)) and the Al content decreases. Similar behavior has already been observed by Badcock et al [15]. Due to its large energy separation with the A transition, we rule out that the $\mathrm{B}$ transition could arise from exciton localization on well-width fluctuations. Moreover, this B emission band is absent from PL spectra measured on homoepitaxial $a$-plane $\mathrm{GaN} /(\mathrm{Al}, \mathrm{Ga}) \mathrm{N}$ QWs grown under the same conditions [26]. Therefore, we can infer that the B emission is specific to heteroepitaxial nonpolar QWs and is likely related to a high density of extended defects, namely BSFs. The optical properties of these localized excitons will be commented on thoroughly in section 3.2.

3.1.3. QW emission broadening versus Al content and well width. Figure 3 and table 1 present the full-width at half maximum (FWHM) of excitonic emission (A transition) at $10 \mathrm{~K}$ for low and high $\mathrm{Al}$ content $\mathrm{QWs}$ as a function of well width. All heterostructures grown along the $a$ axis exhibit a decreasing emission linewidth when the well width is increased from 2 to $7 \mathrm{~nm}$. In polarization-free QWs such as GaAs/(Al,Ga)As (llllll 00 QWs [27], a larger 

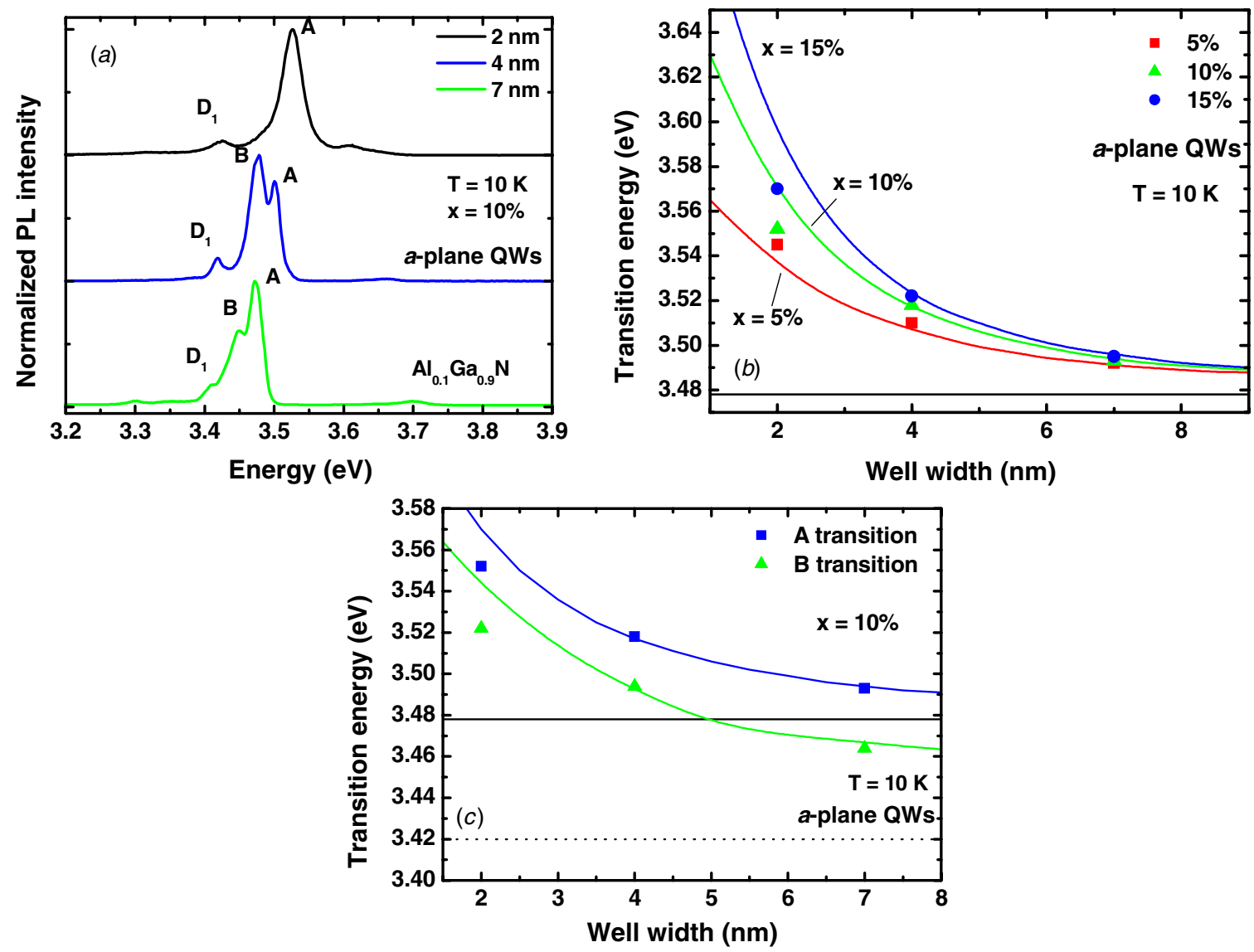

Figure 2. (a) PL spectra measured at $10 \mathrm{~K}$ of $a$-plane $\mathrm{GaN} / \mathrm{Al}_{0.1} \mathrm{Ga}_{0.9} \mathrm{~N}$ QWs. Transitions $\mathrm{A}$ and $\mathrm{B}$ correspond to the recombination of QW excitons localized on well-width fluctuations and on BSFs, respectively. The emission band labeled $\mathrm{D}_{1}$ is related to the BSFs located in the GaN template. (b) $a$-plane $\mathrm{GaN} / \mathrm{Al}_{x} \mathrm{Ga}_{1-x} \mathrm{~N}$ QW emission energy as a function of well width for $\mathrm{Al}$ contents of 5,10 , and $15 \%$, black curves are envelope function calculations. (c) Experimental emission energy of $\mathrm{A}$ and $\mathrm{B}$ emission lines as a function of well width for an $\mathrm{Al}$ content set to $10 \%$ (squares and triangles, respectively). Blue and green curves are results obtained from envelope function calculations for $\mathrm{A}$ and B transitions, respectively. Emission energies of free excitons and excitons bound to BSFs in bulk GaN are also reported (black solid line and dashed line, respectively).

well width is generally associated with a narrower emission line, because $(i)$ well-width variations have a smaller effect on quantized energies and (ii) the wavefunction penetration into the disordered alloy barriers is reduced. In contrast, in $\mathrm{GaN} /(\mathrm{Al}, \mathrm{Ga}) \mathrm{N}$ QWs grown along the $c$-axis, $2.5 \mathrm{~nm}$ thick wells correspond to the best compromise-in terms of emission linewidth-between thin QWs characterized with strong quantum confinement effects and wider ones presenting a large quantum-confined Stark effect. This phenomenon was ascribed to the combined effects of fluctuation of well width and $\mathrm{Al}$ content, on the one hand, and of internal electric field, on the other hand [28]. The latter indeed strengthens, for large well widths, the effect of interface roughness by enhancing the presence probability of carriers at the interfaces and by red shifting the emission energy at these potential fluctuations. The steady narrowing of the emission linewidth with respect to the well width observed for the present $a$-plane QWs is therefore another evidence for the absence of built-in electric field in these structures.

One should also note that in the case of QWs with an $\mathrm{Al}$ content set in the barriers to $5 \%$, the dependence of the emission FWHM versus well width is less pronounced since alloy fluctuations in the barriers are reduced. A difference in strain states between the overgrown and the window regions [29], as well as the presence of extended defects (such as dislocations and stacking faults) that locally relax strain, now plays the most important role in the inhomogeneous broadening of the QW emission.

\subsection{Basal stacking fault emission (B transition)}

3.2.1. Spatial dependence of the $B$ transition. The BSF distribution in our ELO samples is indeed highly inhomogeneous as shown in cross-section TEM images (figure 4(a)). Along the $+c$ direction, above the $\mathrm{SiO}_{2}$ mask, the BSF density is low in the $+c$-wings (about $10^{4} \mathrm{~cm}^{-1}$ ) while we estimate BSF density of $10^{6} \mathrm{~cm}^{-1}$ in the windows. Along the $-c$ direction, a high BSF density is also observed in the $-c$ wings. The low-temperature (LT) $\mu \mathrm{PL}$ spectra of $7 \mathrm{~nm}$ thick $a$ plane $\mathrm{GaN} / \mathrm{Al}_{0.1} \mathrm{Ga}_{0.9} \mathrm{~N}$ and $m$-plane $\mathrm{GaN} / \mathrm{Al}_{0.05} \mathrm{Ga}_{0.95} \mathrm{NQWs}$ are shown in figures $5(a)$ and $(b)$, respectively. They clearly confirm the assignment of the A and B lines to QW excitons and BSF-related QW excitons, respectively. In the highly defective regions, the $\mathrm{B}$ transition indeed dominates the PL spectra to the detriment of the A line. As BSFs propagate 


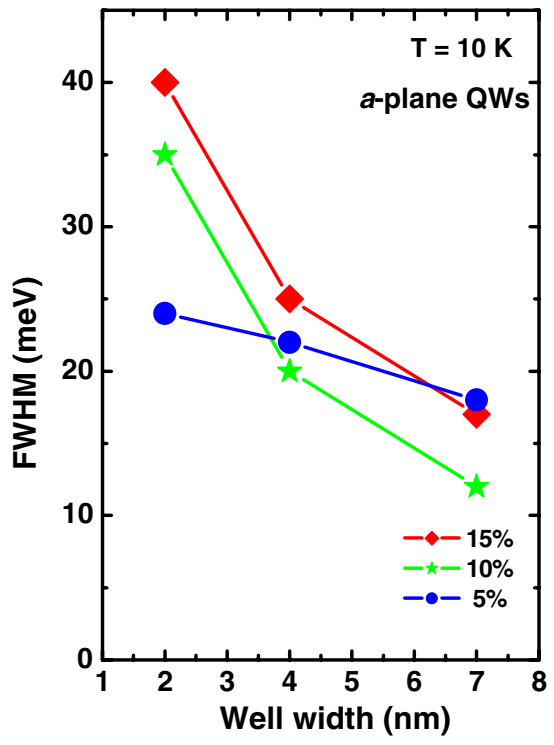

Figure 3. Full-width at half maximum of the A transition emission of $a$-plane $\mathrm{GaN} /(\mathrm{Al}, \mathrm{Ga}) \mathrm{N}$ QWs as a function of well width and $\mathrm{Al}$ content.

from the template to the surface of the sample, going through the QW and the (Al,Ga)N barriers (figure 4(b)), and as the electronic properties of BSFs in low $\mathrm{Al}$ content $(\mathrm{Al}, \mathrm{Ga}) \mathrm{N}$ are likely similar to those in GaN, BSFs should localize excitons in the $(\mathrm{Al}, \mathrm{Ga}) \mathrm{N}$ barriers and in the QW, as they do in bulk $\mathrm{GaN}$. As a consequence, and in agreement with previous PL excitation and cathodoluminescence studies $[15,16]$, we can safely assign the $\mathrm{B}$ transition to the recombination of QW excitons localized on BSFs. Note however that we were not able with our $\mu$-PL set-up to distinguish between regions with bundles of BSFs and those with low-density BSFs, as reported in $[16,30]$.

Where the B line dominates the spectrum (high local BSF density), we observe in the LT $\mu$ PL spectra a weak contribution from BSFs located in the GaN template (3.42 eV line), as well as their counterpart in the $(\mathrm{Al}, \mathrm{Ga}) \mathrm{N}$ barrier, with obviously a localization energy that increases with the $\mathrm{Al}$ content.

Let us finally note that when exciting the $+c$-wings, the QW and the (Al,Ga)N emissions are blue-shifted and redshifted, respectively, compared to the emission from the $-\mathrm{c}-$ wings and the windows. This suggests that in the $+c$-wings, the GaN QWs might undergo a slight compressive strain, whereas the $(\mathrm{Al}, \mathrm{Ga}) \mathrm{N}$ barriers would be under small in-plane dilatation. Given the asymmetry of the system, such strain would most certainly be anisotropic along the $a$ - or the $m$-plane. The presence of such elastic strain in the high quality $+c$-wings is however consistent with the weaker strain relaxation due to the low density of extended defects when using the ELO process.

\subsubsection{Temperature dependence of the $B$ transition.}

Temperature-dependent PL measurements have been performed in order to quantify the localization energy of QW excitons on well-width fluctuations and on BSFs. The evolution with $T$ of the A-line emission energy (figure 6) exhibits the usual S-shaped behavior reported for polar
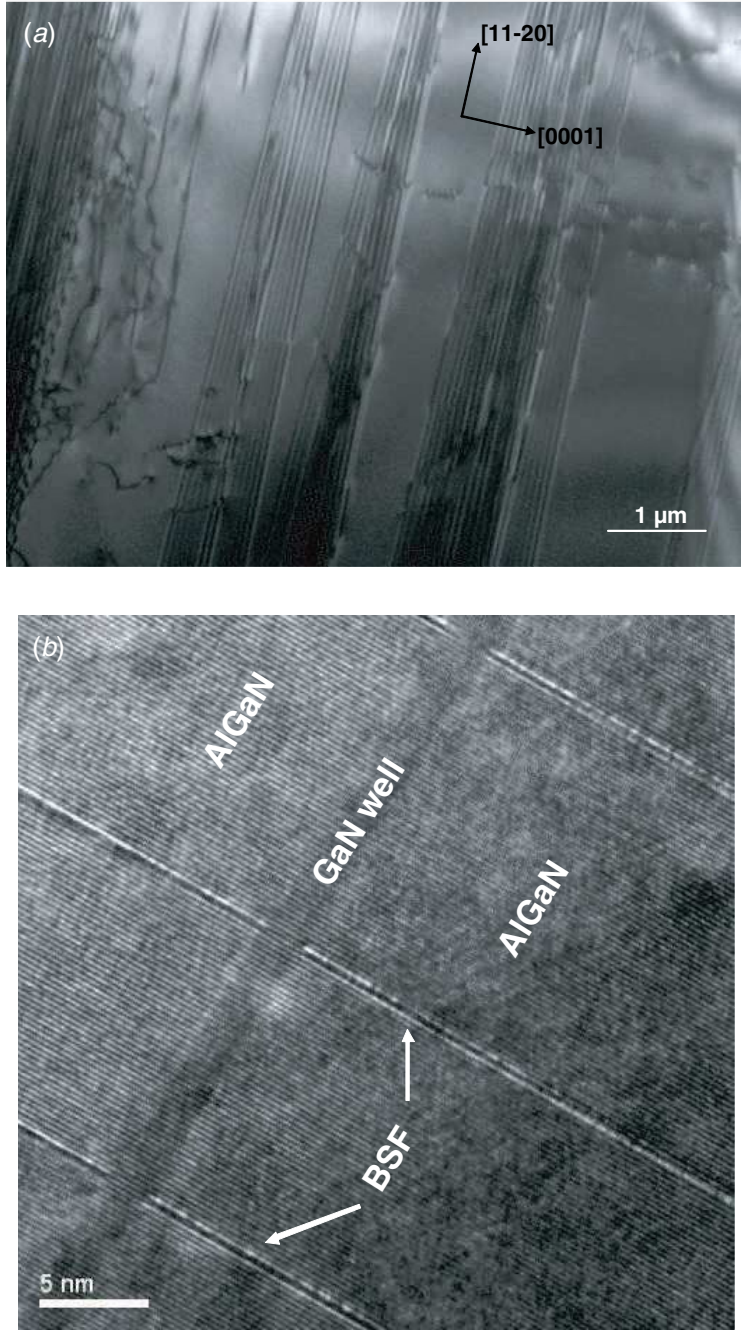

Figure 4. Cross-section TEM images of a $2 \mathrm{~nm}$ thick $a$-plane $\mathrm{GaN} / \mathrm{Al}_{0.1} \mathrm{Ga}_{0.9} \mathrm{~N}$ QW showing $(a)$ the highly inhomogeneous BSF distribution and $(b)$ the propagation of BSFs from the GaN template to the surface, going through the $(\mathrm{Al}, \mathrm{Ga}) \mathrm{N}$ barriers and the $\mathrm{QW}$.

GaN/(Al,Ga)N QWs [23]. At low T, QW excitons are localized on shallow potential fluctuations induced by well-width fluctuations and alloy disorder. When $T$ is increased, carrier delocalization into the whole QW leads to a stable or slightly blue-shifted PL emission (figure 6). At higher $T$, the excitonic emission energy follows the $T$-dependence of the bandgap, resulting in a red-shifted PL. Assuming that excitons are totally delocalized in the two-dimensional QW at $300 \mathrm{~K}$, we performed a fit to the experimental results by using Varshni's semi-empirical formula [31]. We used the same fitting parameters as those given in [32], which, by extrapolation to $T=0 \mathrm{~K}$, allows us to estimate QW exciton localization energies on well-width fluctuations, as shown in figure 6. Such localization energies are reported in table 1 for all samples investigated in this work.

The emission energy of BSF-bound QW excitons also displays an S-shaped dependence with $T$ (figure 6). Previous low-temperature CL study on $a$-plane QWs already revealed 

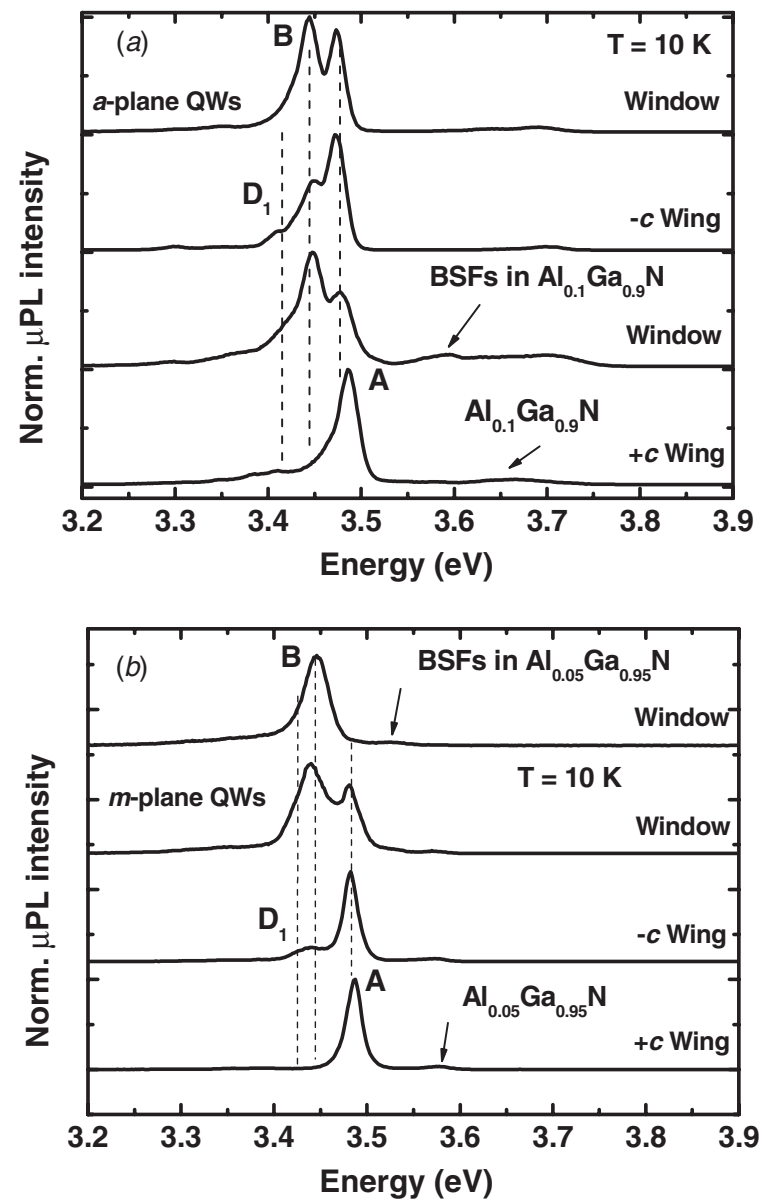

Figure 5. $\mu$-PL spectra as a function of the position of the excitation spot for $(a)$ a $7 \mathrm{~nm}$ thick $a$-plane ELO-GaN/ $/ \mathrm{Al}_{0.1} \mathrm{Ga}_{0.9} \mathrm{~N}$ $\mathrm{QW}$ and $(b)$ a $8 \mathrm{~nm}$ thick $m$-plane ELO-GaN/ $\mathrm{Al}_{0.05} \mathrm{Ga}_{0.95} \mathrm{~N} \mathrm{QW}$. An intense $\mu \mathrm{PL}$ signal from BSF-bound QW excitons is observed in the windows and in the $-c$-wings. A difference in strain state in the $+c$-wings compared to the highly defective regions leads to a blue shifted emission.

intra-BSF localization, which was attributed to well-width fluctuations of the GaN/(Al,Ga)N QWs [16]. However, QW width fluctuation cannot account for the low-temperature redshift observed in figure 6 , and the mechanisms localizing BSFbound QW excitons are therefore definitely more complex. The S-shaped behavior has already been observed for BSFbound excitons in $a$-plane $\mathrm{GaN}[33,34]$ and has been ascribed to the presence of donor nuclei in the vicinity of the BSF planes, as theoretically discussed in [35]. After a careful deconvolution procedure, Varshni fits reveal an intra-BSF localization energy of $33 \mathrm{meV}$ for the $4 \mathrm{~nm}$ wide $a$-plane $\mathrm{GaN} / \mathrm{Al}_{0.05} \mathrm{Ga}_{0.95} \mathrm{~N}$ QW. Intra-BSF localization is consequently stronger in $\mathrm{GaN} /(\mathrm{Al}, \mathrm{Ga}) \mathrm{N}$ QWs than in bulk $\mathrm{GaN}$, where localization energies comprised between 15 and $18 \mathrm{meV}$ have been reported [33,34]. We tentatively attribute the increased intra-BSF binding energy observed in QWs compared to bulk GaN to the combined localization of BSF excitons induced by the QW-width variations [16] and barrier alloy disorder [24]. We do not exclude that donors, with increased binding energies when located in QWs [36], may also efficiently localize BSF-bound QW excitons.

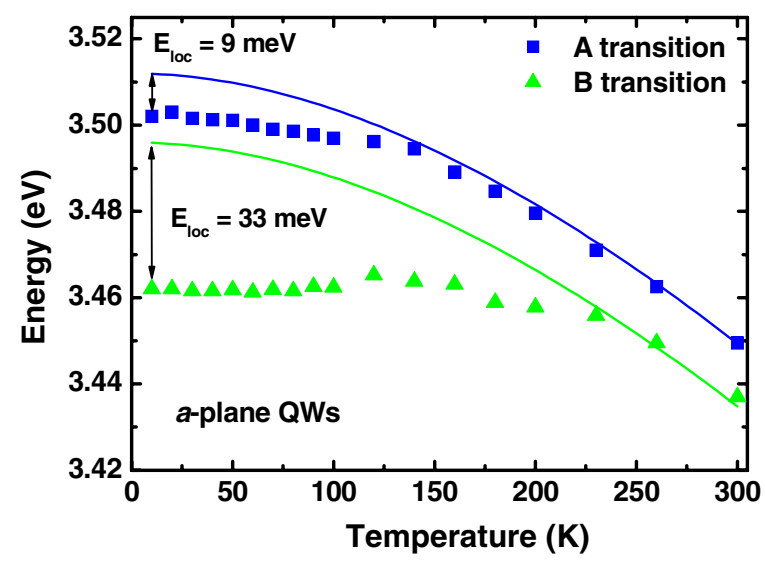

Figure 6. PL emission energy as a function of temperature for A and $\mathrm{B}$ transitions (squares and triangles, respectively) for a $4 \mathrm{~nm}$ thick $a$-plane $\mathrm{GaN} / \mathrm{Al}_{0.05} \mathrm{Ga}_{0.95} \mathrm{~N} \mathrm{QW}$. The two curves are the result of Varshni fits.

\subsection{One-dimensional character of BSF-bound $Q W$ excitons}

3.3.1. Band alignment for $I_{1}$-type BSFs in $\mathrm{GaN} /(\mathrm{Al}, \mathrm{Ga}) \mathrm{N}$ $Q W s$. The energy separation between the emission from $\mathrm{QW}$ and BSF delocalized excitons (figure 2(b) and [15]) is reduced compared to the $45 \mathrm{meV}$ observed in bulk GaN [34]; in a $7 \mathrm{~nm}(2 \mathrm{~nm})$ wide QW, one observes an energy difference of $30 \mathrm{meV}(10 \mathrm{meV})$ between these two transitions. Therefore, BSF excitons are a priori more sensitive to the confinement caused by the $\mathrm{GaN} /(\mathrm{Al}, \mathrm{Ga}) \mathrm{N}$ QW than excitons present in the non-faulted regions of the QW. Experimental and theoretical studies on $\mathrm{I}_{1}$-type BSF in wurtzite $\mathrm{GaN}$ have shown that these extended defects can be seen as shallow three-monolayerwide type-II QWs of cubic-like GaN embedded in a wurtzite matrix [37, 38]. Due to the particular band alignment of $\mathrm{I}_{1^{-}}$ BSFs in wurtzite $\mathrm{GaN}\left(\Delta E_{C}=270 \mathrm{meV}\right.$ and $\Delta E_{V}=-70$ $\mathrm{meV}$ [38]) electrons are weakly confined in the BSF plane while holes are repelled into wurtzite GaN. Consequently, the wavefunction of the resulting type-II exciton exhibits a large spatial extension (figure 7(a)). Our effective potential calculations indeed yield an in-plane pseudo-Bohr radius, $a_{B}$, of $3.9 \mathrm{~nm}$ for the BSF exciton, i.e. an extension $\sim 1.3$ times larger than the exciton Bohr radius in bulk wurtzite GaN. The enhancement of the exciton binding energy in a QW essentially depends on the ratio of the well thickness to the Bohr radius of the non-confined exciton [39]. Therefore, for a given well width, the increase in binding energy for the BSF exciton is larger than for an exciton in defect-free material, explaining in a first approximation the dependences shown in figure 2(c).

As a matter of fact, the case of a QW intersected by a BSF is more complicated, as excitons experience confinement into two orthogonal directions. They are indeed confined along the growth axis by the type-I GaN/(Al,Ga)N QW (figure 7(b)) and

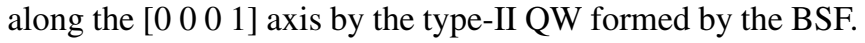
As a result, Badcock et al [15] proposed that the intersection between a BSF and an $a-(m-)$ plane QW results in a quantum wire (QWR) aligned along the $m$ - $(a$-) axis, though clear optical 

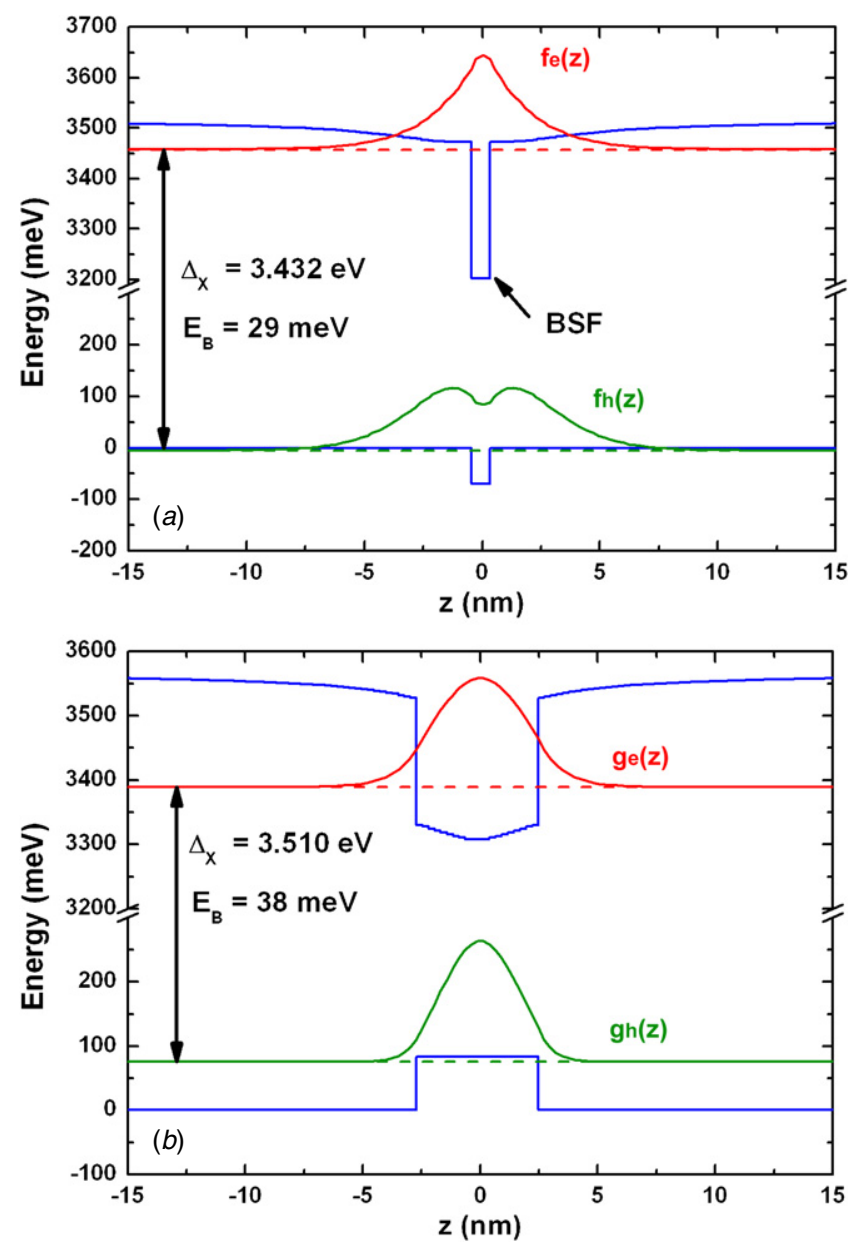

Figure 7. Band profiles (blue) and electron and hole wavefunctions (red and green, respectively) for an exciton confined $(a)$ in an $\mathrm{I}_{1}$-type BSF in wurtzite $\mathrm{GaN}\left(f_{e}(z)\right.$ and $\left.f_{h}(z)\right)$ and $(b)$ in a $4 \mathrm{~nm}$ thick $a$-plane $\mathrm{GaN} / \mathrm{Al}_{0.1} \mathrm{Ga}_{0.9} \mathrm{~N} \mathrm{QW}\left(g_{e}(z)\right.$ and $\left.g_{h}(z)\right) . \Delta_{\mathrm{X}}$ and $E_{B}$ are the calculated exciton emission energy, binding energy and in-plane pseudo-Bohr radius, respectively. evidence of the one-dimensional character of BSF-bound QW excitons is still missing.

The exact calculation of the binding energy and wavefunction of such spatially indirect QWR excitons is complicated and outside the scope of this paper. Nevertheless, we can safely presume that the electron wavefunction is weakly confined along the wire axis, whereas the hole wavefunction is repelled into the wurtzite regions of the QW, as sketched in figure 8. The large extension of the QWR exciton wavefunction would consequently make them sensitive to localization centers located in and away from the BSFs, resulting in the inhomogeneous broadening of the emission observed in figures $2(a)$ and 5.

3.3.2. Time dependence of the $B$ transition. In contrast to what occurs in polar QWs, the oscillator strength and consequently the radiative lifetime of excitons in nonpolar QWs are not expected to depend much on the well width. Still, recent time-resolved cathodoluminescence experiments revealed that the dynamics of excitons in $a$ - and $m$ - plane QWs strongly depend on the local density of BSFs [16]. In the windows and the $-c$-wings, the fast decay of $\mathrm{QW}$ excitons is mainly tributary to their capture by BSFs, while in the $+c$-wings, their decay is essentially of radiative origin. The TRPL decay of nonpolar QWs consequently exhibits a multi-exponential behavior whose slow component most likely accounts for the exciton radiative decay. For each sample, we have reported in table 1 the decay time of the slow component of the QW PL decay. The values range between 230 and 415 ps for the different samples, which we tentatively assign to differences in dislocation density and exciton diffusion length between samples.

Concerning BSF-bound QW excitons, they exhibit at $10 \mathrm{~K}$, in all samples, a slower PL decay than QW unbound excitons (table 1 and figure 9). This results from their deeper localization [40]. The increase of $T$ then simultaneously

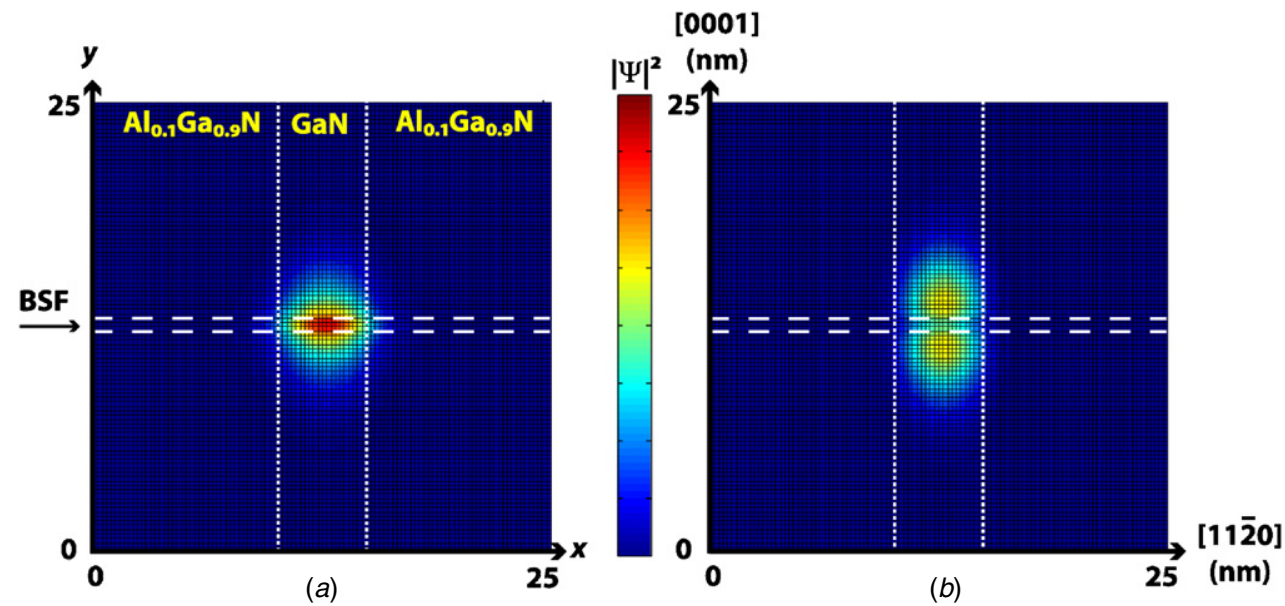

Figure 8. Presumed (a) electron and (b) hole densities of probability $\left(\left|\Psi_{\mathrm{e}}(x, y)\right|^{2}\right.$ and $\left.\left|\Psi_{\mathrm{h}}(x, y)\right|^{2}\right)$ for an exciton confined in a $4 \mathrm{~nm}$ thick $a$-plane $\mathrm{GaN} / \mathrm{Al}_{0.1} \mathrm{Ga}_{0.9} \mathrm{~N}$ QW intersected by an $\mathrm{I}_{1}$-type BSF. The wavefunctions $\Psi(x, y)$ are the result of $\mathrm{N} \cdot g(x) \cdot f(y)$, where $\mathrm{N}$ is a normalization factor and $f$ and $g$ are the calculated wavefunctions shown in figure 7. Dotted and dashed lines represent the interfaces of the QW and the BSF, respectively. 


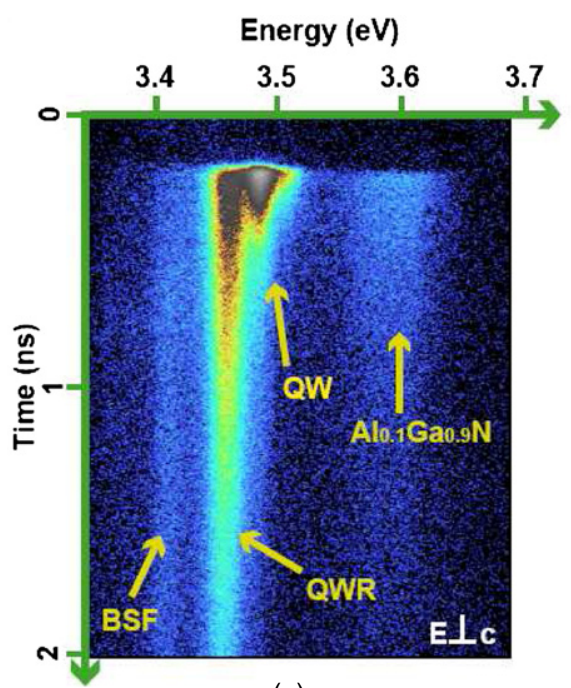

(a)
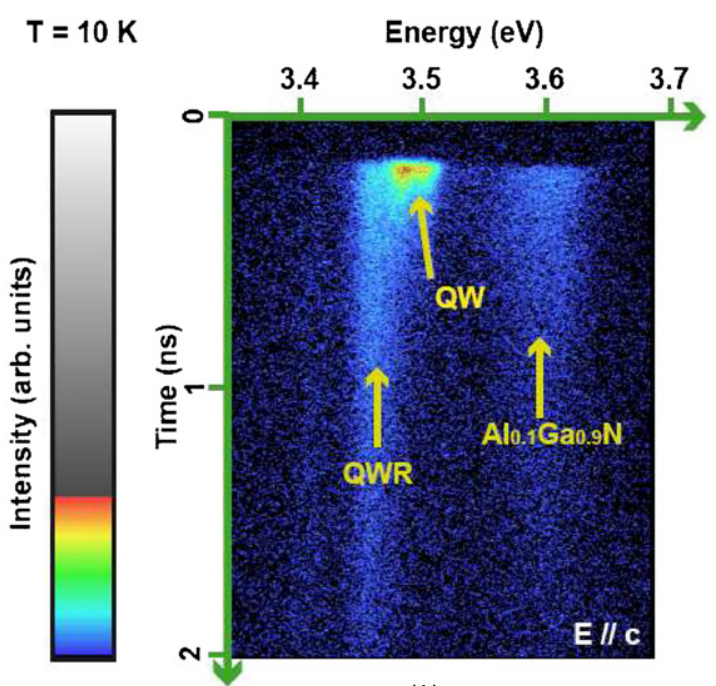

(b)

Figure 9. Time-resolved PL measured at $10 \mathrm{~K}$ of a $4 \mathrm{~nm}$ thick $a$-plane $\mathrm{GaN} / \mathrm{Al}_{0.1} \mathrm{Ga}_{0.9} \mathrm{~N} \mathrm{QW}$ analyzed with a linear polarizer (a) perpendicular and $(b)$ parallel to the $c$-axis. The horizontal and vertical axes correspond to energy and time, respectively. A polarization ratio $\Gamma$ of 0.76 and 0.51 is found for QW and QWR emissions, respectively.

delocalizes excitons into the whole QWR and activates the thermal escape of excitons from the BSFs to the QW. The study of the intrinsic dynamics of BSF-bound QW excitons as a function of $T$ is therefore hindered and one cannot conclude on the 1D character of BSF-bound QW excitons through TRPL experiments [41].

3.3.3. Polarization dependence of the $B$ transition. Yet, QWR emission is also expected to be strongly polarized along the wire axis [42]. For the $a$ - and $m$-plane heterostructures presented in this study, the QWRs resulting from the intersection of the QW and the BSF planes should be aligned along the $m$ - and the $a$-axes, respectively. As a consequence the QWR emission should vanish for light polarization oriented along the $c$-axis. However, the ground state exciton in nonpolar $\mathrm{GaN}$ [43] as well as in shallow nonpolar low $\mathrm{Al}$ content $\mathrm{GaN} /(\mathrm{Al}, \mathrm{Ga}) \mathrm{N}$ QWs is also expected to be 'dark' for polarization along the $\left[\begin{array}{llll}0 & 0 & 0 & 1\end{array}\right]$ direction [44]. Figure 9 shows indeed that both QW and QWR time-resolved emissions are polarized along the $m$-axis. Assuming that the anisotropy for the QW emission originates solely from the crystal symmetry, a stronger polarization anisotropy for BSF-bound QW excitons would underline their 1D character. Defining the polarization degree as $\Gamma=\frac{I_{\perp c}-I_{/ / c}}{I_{\perp c}+I_{/ / c}}$, we observe at $10 \mathrm{~K}$ a stronger polarization anisotropy for BSF-bound QW excitons compared to the QW emission $(\Gamma=0.76$ against 0.51$)$. Thus, the intersection between a BSF and a non-polar QW can indeed be considered as a QWR.

\section{Conclusions}

In conclusion, we have studied the optical properties of $a$ - and $m$-plane $\mathrm{GaN} /(\mathrm{Al}, \mathrm{Ga}) \mathrm{N}$ QWs grown on ELO-GaN templates. The dependence of both QW emission energy and PL linewidth on the Al content and QW width clearly shows the absence of the internal electric field and the high optical quality of our heterostructures. In low Al content QWs, the emission from BSF-bound QW excitons is clearly resolved from the luminescence of a defect-free QW. A qualitative model has been developed to understand the intra-BSF localization of QW excitons observed by means of temperature-dependent photoluminescence measurements. It evidences that the wavefunction of BSF-bound excitons exhibits a large spatial extension and is consequently sensitive to potential localization centers present in and away from the BSF. Finally, polarization-resolved PL has demonstrated the $1 \mathrm{D}$ character of BSF-bound QW excitons, which can therefore be considered as quantum wire excitons.

\section{Acknowledgments}

This work was supported by the Swiss National Science Foundation through Quantum Photonics NCCR and Projects nos 119840 and 122294.

\section{References}

[1] Schmidt M C, Kim K C, Sato H, Fellows N, Masui H, Nakamura S, DenBaars S P and Speck J S 2007 Japan. J. Appl. Phys. 46 L126

[2] Feezel D F, Schmidt M C, Farrell R M, Kim K C, Saito M, Fujito K, Cohen D A, Speck J S, DenBaars S P and Nakamura S 2007 Japan. J. Appl. Phys. 46 L284

[3] Waltereit P, Brandt O, Trampert A, Grahn H T, Menniger J, Ramsteiner M, Reiche M and Ploog K H 2000 Nature (Lond.) 406865

[4] Wang H, Chen C, Gong Z, Zhang J, Gaevski M, Su M, Yang J and Khan M A 2004 Appl. Phys. Lett. 84499

[5] Haskell B A, Wu F, Matsuda S, Craven M D, Fini P T, Denbaars S P, Speck J S and Nakamura S 2003 Appl. Phys. Lett. 831554

[6] Craven M D, Wu F, Chakraborty A, Imer B, Mishra U K, Denbaars S P and Speck J S 2004 Appl. Phys. Lett. 841281 
[7] Paskova T, Paskov P P, Valcheva E, Darakchieva V, Birch J, Kasic A, Arnaudov B, Tungasmitta S and Monemar B 2004 Phys. Status Solidi a 2012265

[8] Sun Y J, Brandt O, Jahn U, Liu T Y, Trampert A, Cronenberg S, Dhar S and Ploog K H 2002 J. Appl. Phys. 925714

[9] Wetzel C, Fischer S, Krüger J, Haller E E, Molnar R J, Moustakas T D, Mokhov E N and Baranov P G 1996 Appl. Phys. Lett. 682556

[10] Rieger W, Dimitrov R, Brunner D, Rohrer E, Ambacher O and Stutzmann M 1996 Phys. Rev. B 5417596

[11] Fischer S et al 1998 J. Cryst. Growth 189/190 556

[12] Rebane Y T, Shreter Y G and Albrecht M 1997 Phys. Status Solidi a 164141

[13] Bandic Z Z, McGill T C and Ikonic Z 1997 Phys. Rev. B 563564

[14] Liu R, Bell A, Ponce F A, Chen C Q, Yang J W and Kahn M A 2005 Appl. Phys. Lett. 86021908

[15] Badcock T J, Dawson P, Kappers M J, McAleese C, Hollander J L, Johnston C F, Sridhara Rao D V, Sanchez A M and Humphreys C 2008 Appl. Phys. Lett. 93101901

[16] Corfdir P, Lefebvre P, Balet L, Sonderegger S, Dussaigne A, Zhu T, Martin D, Ganière J-D, Grandjean N and Deveaud-Plédran B 2010 J. Appl. Phys. 107043524

[17] Zhu T, Martin D, Butté R, Napierala J and Grandjean N 2007 J. Cryst. Growth $\mathbf{3 0 0} 186$

[18] Zhu T, Martin D and Grandjean N 2009 Japan. J. Appl. Phys. 48020226

[19] Haskell B A, Wu F, Craven M D, Matsuda S, Fini P T, Fujii T, Fujito K, DenBaars S P, Speck J S and Nakamura S 2003 Appl. Phys. Lett. 83644

[20] Bougrioua Z, Laügt M, Vennéguès P, Cestier I, Gühne T, Frayssinet E, Gibart P and Leroux M 2007 Phys. Status Solidi a 204282

[21] Vurgaftman I, Meyer J R and Ram-Mohan L R $2001 \mathrm{~J}$. Appl. Phys. 895815

[22] Bellabchara A, Lefebvre P, Christol P and Mathieu H 1994 Phys. Rev. B 5011840

[23] Leroux M, Grandjean N, Laügt M, Massies J, Gil B, Lefebvre P and Bigenwald P 1998 Phys. Rev. B 58 R13371

[24] Gallart M, Morel A, Talierco T, Lefebvre P, Gil B, Allègre J, Mathieu H, Grandjean N, Leroux M and Massies J 2000 Phys. Status Solidi a 180127
[25] Feltin E, Simeonov D, Carlin J-F, Butté R and Grandjean N 2007 Appl. Phys. Lett. 90021905

[26] Teisseyre H, Kaminska A, Franssen G, Dussaigne A, Grandjean N, Grzegory I, Lucznik B and Suski T 2009 J. Appl. Phys. 105063104

[27] Bimberg D, Christen J, Fukunaga T, Nakashima H, Mars D E and Miller J N 1987 J. Vac. Sci. Technol. B 51191

[28] Natali F, Byrne D, Leroux M, Damilano B, Semond F, Le Louarn A, Vézian S and Massies J 2005 Phys. Rev. B 71075311

[29] Bastek B, Bertram F, Christen J, Wernicke T, Weyers M and Kneissl M 2008 Appl. Phys. Lett. 92212111

[30] Corfdir P, Ristić J, Lefebvre P, Zhu T, Martin D, Dussaigne A, Ganière J-D, Grandjean N and Deveaud-Plédran B 2009 Appl. Phys. Lett. 94201115

[31] Varshni Y P 1967 Physica (Utrecht) 34149

[32] Leroux M, Grandjean N, Beaumont B, Nataf G, Semond F, Massies J and Gibart P 1999 J. Appl. Phys. 863721

[33] Paskov P P, Schifano R, Monemar B, Paskova T, Figge S and Hommel D 2005 J. Appl. Phys. 98093519

[34] Corfdir P, Lefebvre P, Levrat J, Dussaigne A, Ganière J-D, Martin D, Ristić J, Zhu T, Grandjean N and Deveaud-Plédran B 2009 J. Appl. Phys. 105043102

[35] Corfdir $P$, Lefebvre $P$, Ristić $J$, Ganière J-D and Deveaud-Plédran B 2009 Phys. Rev. B 80153309

[36] Bastard G 1981 Phys. Rev. B 244714

[37] Salviati G et al 1999 Phys. Status Solidi a 171325

[38] Stampfl C and Van de Walle C G 1998 Phys. Rev. B 57 R15052

[39] Bastard G, Mendez E E, Chang L L and Esaki L 1982 Phys. Rev. B 261974

[40] Rashba E T and Gurgenishvili G E 1962 Fiz. Tverd. Tela (Leningrad) 41029

Rashba E T and Gurgenishvili G E 1962 Sov. Phys. Solid State 4759

[41] Citrin D S 1992 Phys. Rev. Lett. 693393

[42] Maslov A V and Ning C Z 2005 Phys. Rev. B 72125319

[43] Gühne T, Bougrioua $Z$, Laügt $S$, Nemoz M, Vennéguès $P$, Vinter B and Leroux M 2008 Phys. Rev. B 77075308

[44] Leroux M et al 2004 Superlattices Microstruct. 36659

[45] Grandjean N, Damilano B, Dalmasso S, Leroux M, Laügt M and Massies J 1999 J. Appl. Phys. 863714 